\title{
Editorial: Optics and Ultrasound in Biomedicine: Sensing, Imaging, and Therapy
}

\author{
Chao Tian ${ }^{1 *}$, Jun $\mathrm{Xia}^{2}$ and Xueding Wang ${ }^{3}$ \\ ${ }^{1}$ College of Engineering Science, University of Science and Technology of China, Hefei, China, ${ }^{2}$ Department of Biomedical \\ Engineering, University at Buffalo, State University of New York, Buffalo, NY, United States, ${ }^{3}$ Department of Biomedical \\ Engineering, University of Michigan, Ann Arbor, MI, United States
}

Keywords: biomedical optics, ultrasound, medical imaging, photoacoustic imaging, optical coherence tomography, super-resolution imaging

\section{Editorial on the Research Topic}

\section{Optics and Ultrasound in Biomedicine: Sensing, Imaging, and Therapy}

Biomedical optics is a branch of optics that studies the interaction of photon and biological tissues for the purpose of sensing, imaging, and treatment. Since light is fundamentally an electromagnetic wave with an ultra-short wavelength, optical-based sensing, imaging, and treatment technologies have advantages in resolution, contrast, sensitivity, and precision, and, therefore, have found unique applications in a range of biomedical fields. However, these applications are limited to superficial tissues due to the strong scattering of photons. Ultrasound, a mechanical wave with a longer wavelength, has a much greater penetration capability in soft tissues and can reach deep-seated biological targets. As a result, ultrasound-based technologies are widely used and intensively researched in many biomedical applications, ranging from diagnosis, intervention, to therapy. Essentially, biomedical optics and ultrasound both leverage the nature of waves and studies their interactions with biological tissues. Their synergy, such as emerging photoacoustics and photon-medicated ultrasound therapy, opens new possibilities for revolutionary applications in biology and medicine.

This Research Topic gathers and reviews the research community's accomplishments to date on opticaland ultrasound-based sensing, imaging, and therapeutic technologies and novel applications in medicine and biology through 15 contributions by 90 authors across the world. There are seven original contributions on optics-based biomedical research, reporting most recent progress in aberration and image reconstruction in structured illumination microscopy (SIM) (Liu et al.; Luo et al.), multi-wavelength diffuse optical tomography (DOT) (Zhang et al.), hyperspectral and video endoscopy (Cai et al.), femtosecond laser skin interaction (Wang et al.), fiber diffuse speckle based pulse wave measurement (Teng et al.), and backscattering based foodborne pathogens detection and classification (Wang et al.). There are two original contributions on ultrasound-based biomedical research, covering most recent progress in highintensity focused ultrasound (HIFU) based vulvar lichen sclerosus treatment (Qu et al.) and portable ultrasound based food sweetness detection (Zhan et al.). There are four original contributions on the synergy of optics and ultrasound, discussing photoacoustic whole breast imaging (Zhang et al.), photoacoustic sensing of bone chemical composition (Feng et al.), Monte Carlo photon transport simulation in photoacoustic imaging (Lu et al.), and light- and ultrasound-activatable microcapsules for drug delivery (Zhu et al.). In addition, there are two review articles summarizing most recent advances in dual-modal photoacoustic imaging and optical coherence tomography (Hosseinaee et al.) and therapeutic ultrasound-enhanced immune checkpoint inhibitor therapy (Yuan et al.). These contributions encompass fundamental theory, technology developments, biomedical studies and clinical translations of optics and ultrasound in biomedicine and provides a useful snapshot of the fast evolving field that is representative of the international and interdisciplinary scope of interest. 
The topic editors are grateful to the Frontiers in Physics support staff, as well as to the contributors and the reviewers for their invaluable dedication and support.

\section{AUTHOR CONTRIBUTIONS}

All authors listed have made a substantial, direct, and intellectual contribution to the work and approved it for publication.
Conflict of Interest: The authors declare that the research was conducted in the absence of any commercial or financial relationships that could be construed as a potential conflict of interest.

Copyright $\odot 2021$ Tian, Xia and Wang. This is an open-access article distributed under the terms of the Creative Commons Attribution License (CC BY). The use, distribution or reproduction in other forums is permitted, provided the original author(s) and the copyright owner(s) are credited and that the original publication in this journal is cited, in accordance with accepted academic practice. No use, distribution or reproduction is permitted which does not comply with these terms. 